\title{
Comparative Study of Binding Behaviour of Cu(II) with Humic Acid and Simple Organic Compounds by Ultrasound Spectrometry
}

\author{
Martina Klucakova* \\ Centre for Materials Research, Faculty of Chemistry, Brno University of Technology, Purkyňova 118, 61200 Brno, \\ Czech Republic
}

\begin{abstract}
Several simple organic (hydroxy)acids as models of principal functional moieties of humic acids and the copper ion as a model ion were used to investigate the metal-humic interactions. The quantum chemical calculations have been performed showing differences in the interaction strength and stability of the metal-acid complexes formed by different aromatic mono- and polycarboxylic acids and their hydroxyl-containing analogues. In this study, new results from the high resolution ultrasound spectroscopy will be presented. The ultrasound propagation velocity, which is sensitive to the intermolecular interactions, has been measured during titration of the models by solution of the copper(II) salt. The dependence of ultrasound velocity on added amount of the copper(II) ions enabled to follow the metal interactions with the individual functional groups and to elucidate the effect of structure of complexing molecule on its binding with the metal. Changes in the slope of the dependence were used to find the saturation of binding and to follow changes of hydration of interacting species. The differences observed for the individual models show that there are active centers not only with various strength and stability of the formed complexes but also with their various rigidity and ability of conformational changes.
\end{abstract}

Keywords: Humic acid, heavy metal, model compound, ultrasound spectrometry.

\section{INTRODUCTION}

Metal binding by humic acids isolated from various sources is the subject of many studies. [1] published a thorough study on metal binding by solid peat and soil humic acids and its tightness as revealed by water and acid washing. It was demonstrated with ten metals that the tight metal binding capacities of humic acids from different origins are similar under the same loading conditions. The comprehensive investigation of adsorption of the metal ions by humic acids extracted from brown coals was published by Martyniuk and Wieckowska [2]. It also included a brief review of the literature data on this problem. The main conclusion was that although the affinity of humic acids of various origins for various heavy metals is indisputable, opinions on the structures of metal-humic complexes and the involvement of various functional groups in them are not unified. There are differences in published comparative series of adsorption capacities for various metals. The experimental data showed that the brown coal humic acids have the highest affinity for $\mathrm{Pb}, \mathrm{Ag}, \mathrm{Hg}, \mathrm{Cu}, \mathrm{Ba}$ and $\mathrm{Cd}$ ions from 17 metals tested and that metal binding is realized mainly through carboxylic and phenolic groups. $\mathrm{Pb}, \mathrm{Ag}, \mathrm{Hg}$ and $\mathrm{Cu}$ ions are able to interact with phenolic groups, even at low $\mathrm{pH}$.

The utilization of model compounds for study of the humic acids reactivity is relatively common. Saar and Weber

*Address correspondence to this author at the Centre for Materials Research, Faculty of Chemistry, Brno University of Technology, Purkyňova 118, 61200 Brno, Czech Republic; Tel: +420541149410;

Fax: +420541149398; E-mail: klucakova@fch.vutbr.cz
[3] utilized salicylic acid as a standard for study of complexation properties of copper(II) with humic substances. Antonelli et al., [4] used simple organic molecule as potassium phthalate, resorcinol and maleic acid for the calorimetric investigation of formation of humic complexes. Cariati et al., [5] studied 2,6-dimethoxybenzoic acid as the humic-like model for complexation with $\mathrm{Mn}, \mathrm{Fe}, \mathrm{Co}, \mathrm{Ni}, \mathrm{Zn}$ and $\mathrm{Cu}$ by thermal analysis. The thermal stability order $\mathrm{Ni}>\mathrm{Co}>\mathrm{Fe}>\mathrm{Yn}>\mathrm{Mn}>\mathrm{Cu}$ was obtained by comparing the dehydration temperatures of the complexes. Micera et al. [6] concluded that metal ions are directly bound to the carboxylic groups and no hydrogen bonding takes place in this case. Borges et al., [7] studied the complexation of copper(II) with various phenolic acids as the ligand models of humic acids. They observed no significant differences in the complexation properties of their models towards copper(II). Because all their models contained the catechol group, they postulated that the complexation process is intrinsically related with its presence. Murray and Linder [8, 9] devised a model of fulvic acids based on randomized positioning of the functional groups and aromatic rings. Their results showed that the predominant bidentate sites are likely to be phthalate- and salicylate-type sites with a significant proportion of the aromatic carboxylic and phenolic groups not participating in the chelating sites. They determined that the affinity of metals for fulvic acids decreases in the order $\mathrm{Fe}(\mathrm{III})>\mathrm{Cu}(\mathrm{II})>\mathrm{Zn}$ (II) $>\mathrm{Mn}$ (II) $>$ $\mathrm{Ca}(\mathrm{II})>\mathrm{Mg}(\mathrm{II})$. Bryan et al., [10] applied their model to both humic and fulvic acids and added several models included the nitrogen atom. The important role of $\mathrm{N}$ content in the affinity of humic substances towards copper was predicated also by Gregor et al., [11]. 
Other authors [12, 13] reported that there are four possibilities of humic binding with metal ions: 1) chelation between carboxylic and phenolic groups, 2) chelation between two carboxylic groups, 3) complexation with carboxyl group and 4) binding with phenols or phenolic ethers.

Humic acids isolated from South-Moravia lignite are used in this work. The most distinct parameter of the lignite humic acids is the low nitrogen content followed by the high carbon content and the high ratio of aromatic to aliphatic carbon. The lignite humic acids contain high amounts of aromatic structures with the oxygen rich groups giving them the high affinity to metal ions. In view of low nitrogen content and high aromaticity of the used humic acids, this work is focused on the models with the aromatic structure and the carboxylic and phenolic groups. Pyrocatechol, phthalic and salicylic acids were used both for the variety of the most important functional groups in ortho-position both for relatively high content of aromatic structures in lignitic humic acids. The new band in UV/VIS spectrum was observed after the the complexation of hydroquinone with copper [14] in spite of the less advantageous para-position of phenolic groups and the lower efficiency of the reaction predicted from computed reaction enthalpy [15] EDTA and citric acid were used as "classical" ligands for the metal ions.

Copper is a traditional model ion to study the metalhumic interactions due to its strong affinity for the binding sites of humic acid.

Comparing the results of quantum chemical calculation for various metals [15], copper(II) was determined as the metal ion with the highest stability of its complexes with humic models. Therefore it was used for investigation of the metal-humic models interactions in this work.

The main aims of this study are the introducing high resolution ultrasound spectrometry into investigation of complexation properties of humic acids and the utilization of measured ultrasound parameters for the determination of the saturation of binding and to follow changes of hydration of interacting species. The high resolution ultrasound spectrometry is a novel technique based on the measurements of parameters of ultrasonic waves propagating through samples. Due high resolution mainly in determination of ultrasound velocity the method is sensitive to, e.g., details of molecular organization or solvating state. The measured ultrasound propagation velocity is very sensitive to the intermolecular interactions and can be used to follow the metal interactions with the individual functional groups of humic acids and the model compound and to elucidate the effect of structure of complexing molecule on its binding with the metal. Changes in the slope of the dependence has been used to find the saturation of binding and to follow changes of hydration of interacting species.

\section{MATERIALS AND METHODS}

Humic acids were isolated from South Moravia lignite by standard alkaline extraction. Lignite was stirred with the extractant $\left(0.5 \mathrm{~mol} \mathrm{dm}{ }^{-3} \mathrm{NaOH}+0.1 \mathrm{~mol} . \mathrm{dm}^{-3} \mathrm{Na}_{4} \mathrm{P}_{2} \mathrm{O}_{7}\right)$ in the $20 \mathrm{~g}$ per $1 \mathrm{dm}^{3}$ ratio for 12 hours. The formed suspension was kept overnight and the next day the solution was separated from the solid phase and acidified by $20 \%$ hydrochloric acid to the $\mathrm{pH}$ below 1 . The solid residue was put into another $1 \mathrm{dm}^{3}$ of the extractant and after 1 hour of stirring the extract was separated and acidified in the same way. The acid extracts were kept in the refrigerator overnight. The precipitated humic acids were purified using the $\mathrm{HCl} / \mathrm{HF}$ mixture $(48 \mathrm{~h})$, washed repeatedly by the deionized water until free from chlorides and dried in oven at $50{ }^{\circ} \mathrm{C}$. More details on the chemical structure of the initial lignite matrix, as well as that of the isolated humic acids, can be found in previous works [16, 17]. The elemental composition and total acidity of the used humic sample are listed in Table $\mathbf{1 .}$

Humic sol and solutions of the model compounds for the ultrasonic study were prepared by the dissolution of humic acids in $\mathrm{NaOH}\left(0.1 \mathrm{~mol} \mathrm{dm}^{-3}\right)$ and the addition of the same

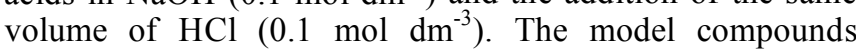
(citric, salicylic acids, EDTA, pyrocatechol, and hydroquinone) were dissolved in the water or in the $\mathrm{NaCl}$ solution (in order to obtain the same ionic strength as in case of the humic sol). The content of acidic groups in the humic sol and the solution of model compounds was $62 \mu \mathrm{mol} \mathrm{dm}{ }^{-3}$.

The ultrasonic spectrometer with high resolution HR-US 102 (Ultrasonic Scientific, Ireland), was utilized for the measurement of basic ultrasonic parameters at three various frequencies $(5110,8220$ and $12200 \mathrm{kHz})$. No changes of the ultrasonic parameters on the applied frequency were occurred and the presented results are the average values of these three determinations. The resolution of the spectrometer used for measuring ultrasound parameters is $10^{-}$ $5 \%$ for the velocity and $0,2 \%$ for the attenuation. The device consists of two independent cells tempered at $25{ }^{\circ} \mathrm{C}$. Both cells were filled by the same model compound $\left(1 \mathrm{~cm}^{3}\right)$. The sample in cell 1 was titrated by the $\mathrm{CuCl}_{2}$ solution $(1 \mathrm{mmol}$ $\mathrm{dm}^{-3}$ ) up to the saturation of acidic groups in the model. The ultrasonic velocity $(U)$ and attenuation $(N)$ in both cells was measured, the the resulting values of $\Delta U$ and $\Delta N$ were calculated as the differences between the sample and reference cells.

\section{RESULTS AND DISCUSSION}

The reaction enthalpies $\left(\Delta H_{\mathrm{r}}\right)$ computed for various $\mathrm{Cu}$ complexes related to the model compounds used in this work are listed in Table 2. The semiempirical method of the INDO method of molecular orbitals parameterized for the $\mathrm{Cu}$ complexes was used for calculation of electron structure. The

Table 1. Elemental Composition (Normalized on Dry Ash-free Sample), Total Acidity and Ash Content of Used Lignitic Humic Acid

\begin{tabular}{|c|c|c|c|c|c|c|}
\hline $\begin{array}{c}\mathrm{C} \\
(\text { at. \%) }\end{array}$ & $\begin{array}{c}\mathrm{H} \\
\text { (at. \%) }\end{array}$ & $\begin{array}{c}\mathbf{N} \\
\text { (at. \%) }\end{array}$ & $\begin{array}{c}\text { S } \\
(\text { at. \%) }\end{array}$ & $\begin{array}{c}\text { O } \\
\text { (at. \%) }\end{array}$ & $\begin{array}{c}\text { Total Acidity } \\
\left(\mathbf{m m o l} \mathbf{g}^{-\mathbf{1}}\right)\end{array}$ \\
\hline \hline 43.93 & 40.18 & 0.72 & 0.22 & 14.96 & 4.94 \\
\hline
\end{tabular}


Table 2. Reaction Enthalpies $\Delta H_{\mathrm{r}}$ Computed for Cu-model Complexes $\left({ }^{a}\right.$ in Acidic Medium, ${ }^{b}$ in Alkaline Medium)

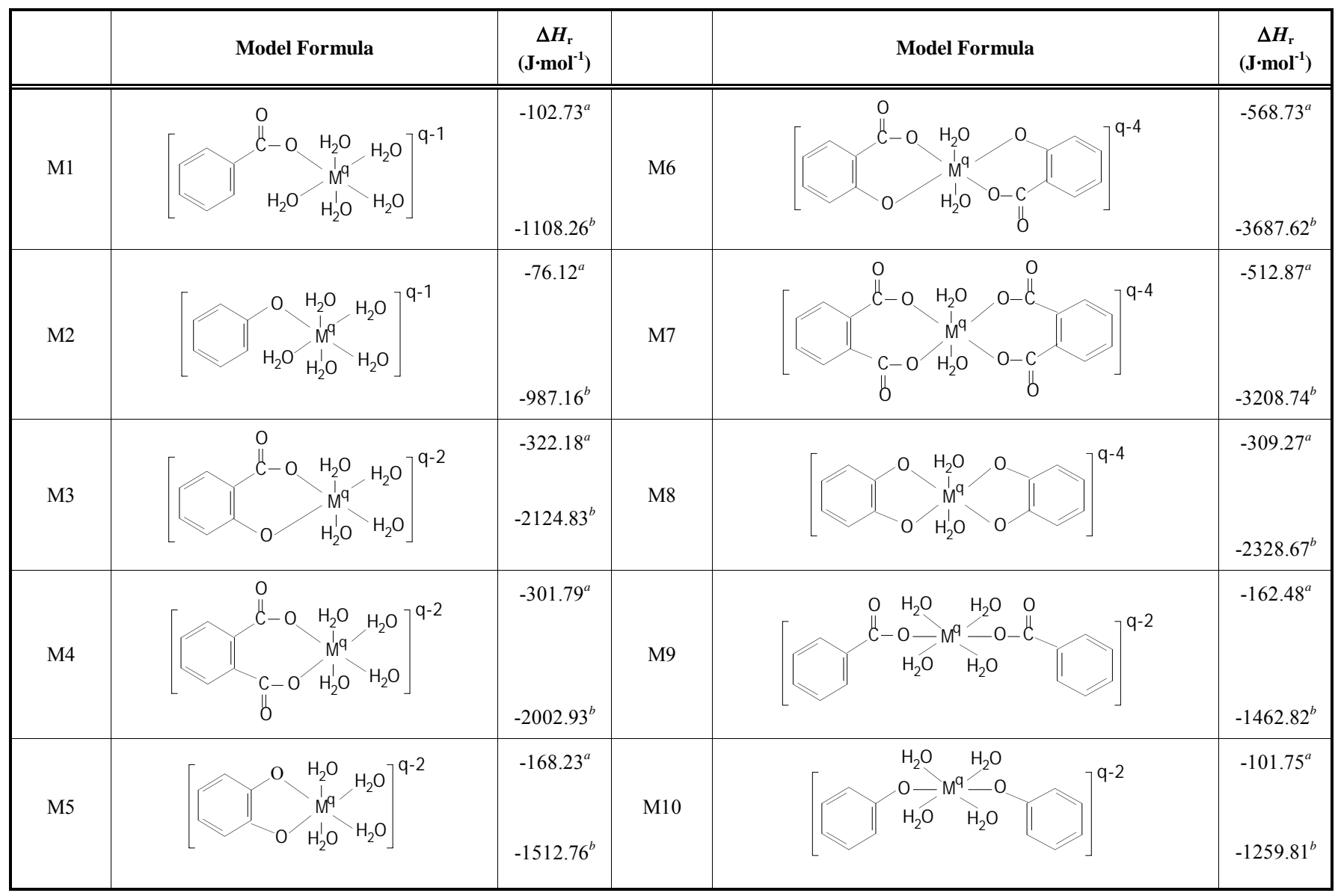

parametrization $\mathrm{MM}+$ was used for the optimization of molecular geometry. The complexes were assumed in the form of hydrates. The INDO method provided the values of total energies of reacting species and reactions entalpies listed in Table 2. The details as well as the results obtained for other metal complexes can be found in ref. [15]. The reaction enthalpies obtained by means of the quantum chemistry calculations are utilized here as a parameter for predicting affinity of the individual binding sites in humic acids to the metal ions. We can see that all models give the negative values of $\Delta H_{\mathrm{r}}$, which show on the good affinity of the models to copper(II). Because the lower values of $\Delta H_{\mathrm{r}}$ correspond with their higher affinity to the copper(II) ions, the order of stability of the model complexes according the $\Delta H_{\mathrm{r}}$-values is: M6 $>\mathrm{M} 7>\mathrm{M} 8>\mathrm{M} 3>\mathrm{M} 4>\mathrm{M} 5>\mathrm{M} 9>$ $\mathrm{M} 10>\mathrm{M} 1>\mathrm{M} 2$. It means that the most preferable models should be the bi-molecular structures with four functional groups, followed by the mono-molecular complexes with two functional groups in the ortho-position. All these structures make possible the chelating effect., which is stronger for the six-member rings and the carboxylic groups. The results published in our previous works [14, 18] confirmed the very high affinity of salicylic acid (corresponding with models M3 and M6) to the copper(II) ions and the high reaction rate in comparison with the other models.

The ultrasound spectrometry is moreover able to show the saturation in binding of the used ligands and metal ions.
The method is based on observing interactions of the ultrasound wave with the studied system. Two principal characteristics are measured - the ultrasound velocity and the attenuation (decrease in the amplitude). The velocity reflects the local elasticity and density of the material, which are determined by the molecular arrangements, conformation and the solvation shell. The attenuation reflects in homogeneous samples the fast relaxation (chemical) processes and in the heterogeneous samples scattering of the ultrasound wave, which can be used for particle sizing. Due the high resolution mainly in determination of the ultrasound velocity the method is sensitive to, e.g., details of the molecular organization or the solvating state. Applications thus may cover the study of micellization or aggregation, ligand-ligate binding, metal ion-(bio)polymer interaction, conformation changes etc. [19-21]. Because both cells of the ultrasaund spectrometer were filled by the same model compound (or humic sol) and only the solution in one cell was titrated, the obtained difference between the ultrasound velocity or the attenuation in the sample and reference cells represent only the contribution of added titration agent in this case. Changes in the slope of the concentration dependence thus could be used to find the saturation of binding and to follow changes of the hydration of interacting species. The results obtained for salicylic acid are shown in Fig. (1). The $\alpha$-value ( $x$-axis) is the ratio between added copper(II) ions and the total amount of functional groups $(\mathrm{COOH}$ and $\mathrm{OH})$ of the model 


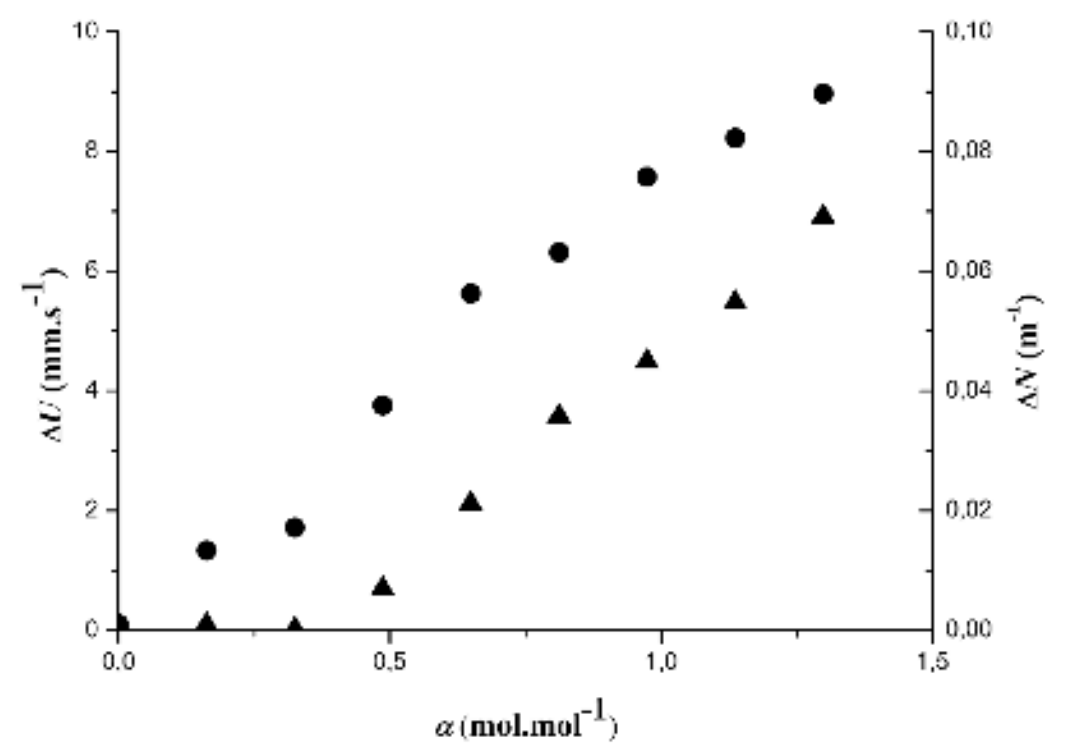

Fig. (1). Results of ultrasound titration of salicylic acid by $\mathrm{CuCl}_{2}(\Delta U-\operatorname{circles,} \Delta N-$ triangles).

compound in the titrated solution. This ratio therefore represents the degree of saturation, but it is necessary to take in consideration that the bivalent copper(II) were used. Copper is able to form complexes with various numbers of ligands and $\alpha$-value is the dergee of saturation of their functional groups.

We can see that titration of the model causes gradually the increase of $\Delta U$ and $\Delta N$ but the concentration dependence is not linear. Because properties of the model solution in the reference cell do not change, the difference in $\Delta U$ and $\Delta N$ reflect only the influence of the titration. The ultrasound velocity $U$ is the function of density and compressibility. The increase of both these quantities causes the decrease of the ultrasound velocity, therefore the data in Fig. (1) can be influenced by the dilution or by the growth of sample rigidity. In order to study the problem in detail, we titrated the sample in one cell by water and also with the $\mathrm{NaCl}$ solutuion. It was found that the simple dilution in this concentration range cannot cause the observed changes in slopes both in case of the salicylic acid and in other samples. The most important factor is therefore the compressibility or its changes in titration by copper(II) salt. As we can see in Fig. (1), the main changes in $\Delta U$ and $\Delta N$ for titration of the salicylic acid were observed around $\alpha=0.5$. This value means that each copper(II) ion is bonded by two acidic functional groups. It agrees with structure M3 in case of the salicylic acid. The increase of the attenuation for $\alpha>0.5$ and the change in slope of the velocity dependence are connected with the electrostatical neutralisation and the saturation of model binding sites. The local extreme in the region of lower $\alpha$-values is probably caused by intermediate forming structure M6. Although the computed reaction enthalpy for M6 is more favorable in comparison with M3, the stability of formed complexes is influenced by their mobility. It is probable that the bi-molecular complex can more easily dissociate or go over to the more rigid mono-molecular complex M3. It was found that the other model compounds with functional groups in ortho-position (phthalic acid and pyrocatechol) have the similar behaviour in titration by
copper(II) ions as the salicylic acid. The saturation of functional groups of both these compounds were observed at $\alpha=0.5$, with the less obvious changes around $\alpha=0.25$. Therefore these models form the mono-molecular structures M4 and M5 with the intermediate forming structures M7 and M8 which are gradually destroyed by further addition of copper(II) ions. The higher affinity of carboxylic groups of the phthalic acid caused that the stabilization of the measured values $\Delta U$ and $\Delta N$ after each copper(II) adding was much faster in comparison with the pyrocatechol.

The chelating effect cannot be applied in case of the functional groups in meta- or para-positions for the spatial arrangement, therefore the result of the ultrasound titration of hydroquinone which has phenolic groups in the paraposition are different (see Fig. 2). We can see that the shave of observed dependencies $\Delta U$ and $\Delta N$ is very similar. The observed changes in slopes around $\alpha=1$ are indicative of forming structure M2, thus each functional group are bonded with one copper(II) ion. The less marked change in slope of the $\Delta U$ dependency at $\alpha=0.5$ implies that the structure M10 with the more favourable computed interaction enthalpy (Table 2) can be formed, but the lower affinity of copper(II) ions to the phenolic functional groups and the higher mobility of the bi-molecular complex caused its easy decomposition. In our previous works [14, 18], we measured the UV/VIS spectra of all used model compounds and their complexes with copper. The new band was observed also in UV/VIS spectrum of the hydroquinone after addition of copper(II) which confirmed the complex forming.

The results of ultrasound measurement obtained for the humic acids are shown in Fig. (3). We can see that (in contrast to the used model compounds) the $\Delta U$-value decreases with the addition of copper(II) ions and the obtained concentration dependence has a relatively marked minimum. The minimum was determined for the $\alpha$-value slightly above 0.5 , which corresponds with the hypothesis that the bivalent ions are bonded by two acidic functional groups. The very low ultrasound velocity at $\alpha=0.25$ in 


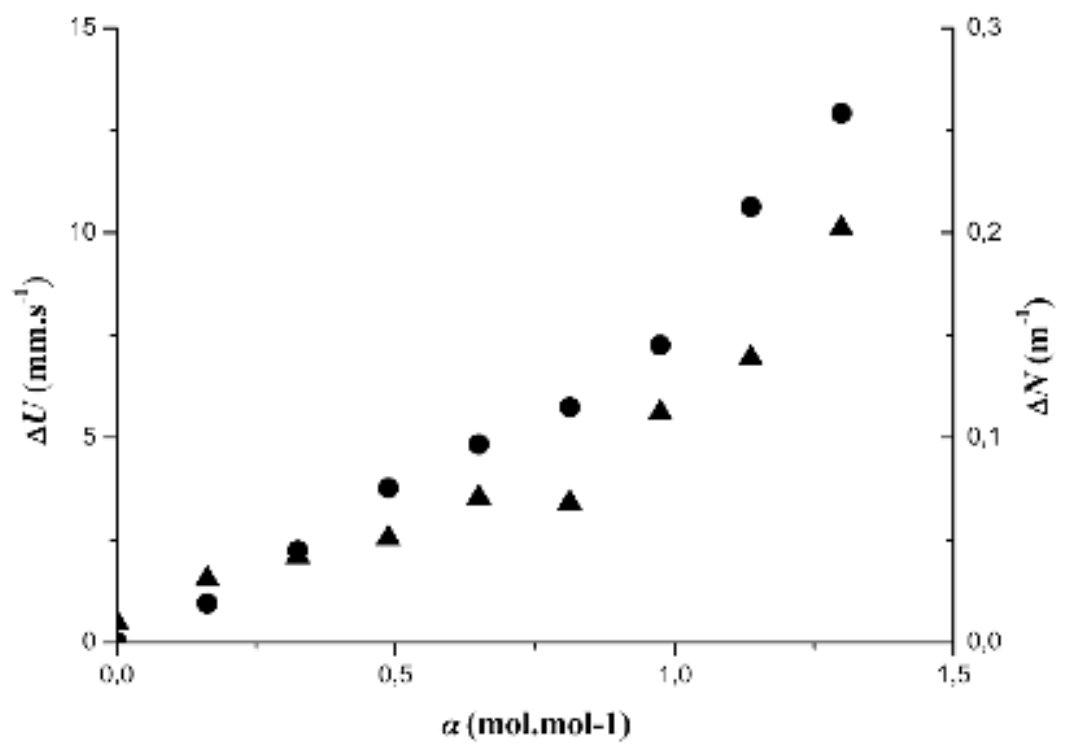

Fig. (2). Results of ultrasound titration of hydroquinone by $\mathrm{CuCl}_{2}(\Delta U-\operatorname{circles}, \Delta N-$ triangles).

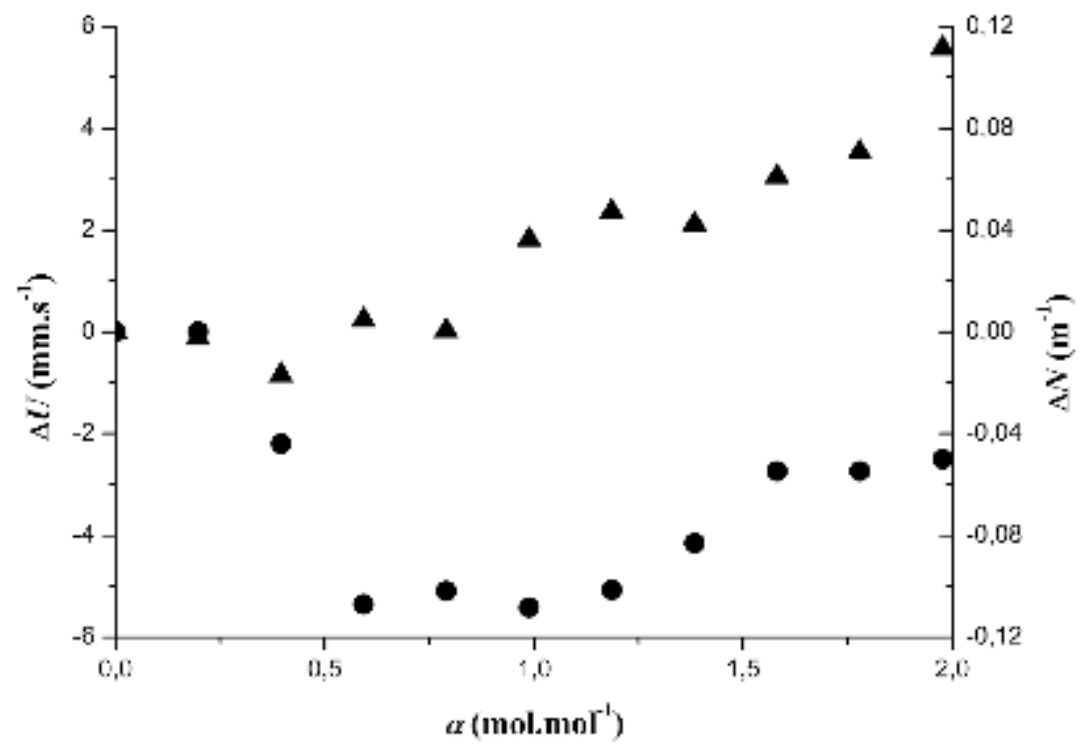

Fig. (3). Results of ultrasound titration of humic acid by $\mathrm{CuCl}_{2}(\Delta U-$ circles, $\Delta N-$ triangles $)$.

comparison with the model compounds shows that only little bi-molecular structures like M6, M7 and M8 are formed. The reasons are probably the spatial arrangement and formation of the strained cycles in this arrangement. The formation of copper-humic complexes results in the initial decrease of ultrasonic velocity. It is caused by the release of hydration water from the coordination shell of copper and atomic groups of humic acids. The compressibility of water in the hydration shells of the ligand is less than the compressibility of bulk water therefore transferring of hydration water into the bulk water increases the total compressibility of the solution, thus reducing the ultrasonic velocity. When all available sites are occupied the curve levels off. At higher concentrations of copper the electrostatically neutralised humic molecules begin to form the aggregates, which could cause the increase of $\Delta U$ at this stage. Pandey et al., [13] published various degree of the saturation of humic acids in the dependence of its origin and experimental circumstances. They determined that their soil humic acids form the complexes with copper at $\alpha=0.5$ or 1 .

The reaction enthalpy between humic acids and copper(II) ions was determined previously in ref. [14]. The value obtained experimentally by means of the isothermal titration calorimetry is $\Delta H_{\mathrm{r}}=-1258 \mathrm{~J} \mathrm{~mol}^{-1}$. This value is less negative than the enthalpies computed for the most favorable structures (see Table 2). It shows that also less suitable binding sites can take place in the interactions of humic acids. The complex structure of humic acids gives many possibilities for the metal ions complexation with various strength of bonds. Our kinetic study of humic complexation [22] showed that the interactions between humic acids and copper(II) ions are, in spite of the high affinity, relatively slow. The high affinity causes that copper(II) ions very 
quickly occupy the majority of humic binding sites in first step of complexation. Because the copper(II) ions need two functional groups to form the stable $\mathrm{Cu}$-humic complexes, it is necessary to find them not too far from each other. The functional groups that cannot be bonded by copper(II) ions due their spatial arrangement can reconnect with the hydrogen ions (reverse reaction of the first step). This rearrangement of the formed complexes causes the increase of conductivity (liberation of copper(II) ions) up to achievement of equilibrium. The minimum on $\mathrm{pH}$ curve and the slow stabilization of conductivity are typical for this twostep complexation mechanism [22]. The results obtained from corresponding measurements for the model compounds are similar, if the chelating effect is not too strong $[14,18]$. The model compounds as 1,4-benzenediol give similar kinetic data as in the case of humic acids. On the other hand, the complexation of salicylic acid was quicker and no $\mathrm{pH}$ minimum was observed in data of kinetic measurements. The high affinity of salicylic acid to copper(II) ions and the strong chelating effect cause probably that no re-arrangement is required in this case.

The results of ultrasound titration for citric acid (as one of the frequently used complexation agent) are shown in Fig. (4). According the obtained $\Delta U$ and $\Delta N$ dependencies one copper(II) ion is coordinated with two or three functional groups in this case. Many authors described copper citrate as the complex with ratio $\mathrm{Cu}$ :ligand 1:1. On the other hand, others described more possible structures of formed complexes. Maketon and Ogden [23] published that citric acid is able to form up to five different complex species in the presence of copper(II) ions. Field et al. [24] published that the copper citrate exists as the monomer only at low and high $\mathrm{pH}$ values, whilst over an intermediate range of $\mathrm{pH}$ a dimeric species are formed. The binuclear copper citrate complexes were observed also in [25].

Ivanov and Tsakova [26] published the structure of copper citrate complex as copper(II) ion bonded with three $\mathrm{O}$ donor atoms and one water molecule as ligand. The copper forms the six-member cycle with one hydroxyl group and one carboxylic one and the five-member cycle including the same hydroxyl but other carboxylate. One carboxylic groups remains free in this case and it is able to bind an additional copper(II) ion. Natter and Hempelmann [27] concluded that the citric acid has several possibilities for the complex formation and its hydroxide group is able to form the hydrogen bonds. All these facts can be reflected in our observations. The formation of complex and the rearrangement in its structure cause probably the expression of water from the hydration shell, which causes the decrease of compressibility compensated partially by the density increase. It is interesting that so called constant region in the concentration dependence of $\Delta U$ begins if one copper(II) ion falls on two functional groups of citric acids. It could mean that the gradual addition of copper(II) ions can cause the formation of complexes in ratio $\mathrm{Cu}$ :ligand $=2: 1$. The constant region then implies some re-arrangement in the structure including the formation of dimers.

The results obtained for EDTA are similar to citric acid. Contrary of the citric acid the "constant region" begins at $\alpha=0.25$. EDTA forms the complexes with metal ions usually in molar ratio 1:1, independently on its valence [28], but formation of the binuclear $\mathrm{Cu}$-EDTA complexes in ratio 2:1 was also published [29]. Our results indicates that the change of slope in the concentrations dependence of $\Delta U$ corresponds with the ratio one copper(II) ion and four functional groups (which is ratio $\mathrm{Cu}$ :EDTA $=1: 1$ ). Other change of the slope was observed at $\alpha>0.5$. This increase of the ultrasound velocity could be connected with the formation of binuclear complexes, which are much less soluble and are formed at the excess of copper(II) ions in weakly acidic solutions as was described in [29]. The published value of enthalpy of reaction of EDTA with copper(II) ions is $-34.3 \mathrm{~kJ} \mathrm{~mol}^{-1}$ [30], which is more negative value than the values computed for the other used models (Table 2). It could imply EDTA as the

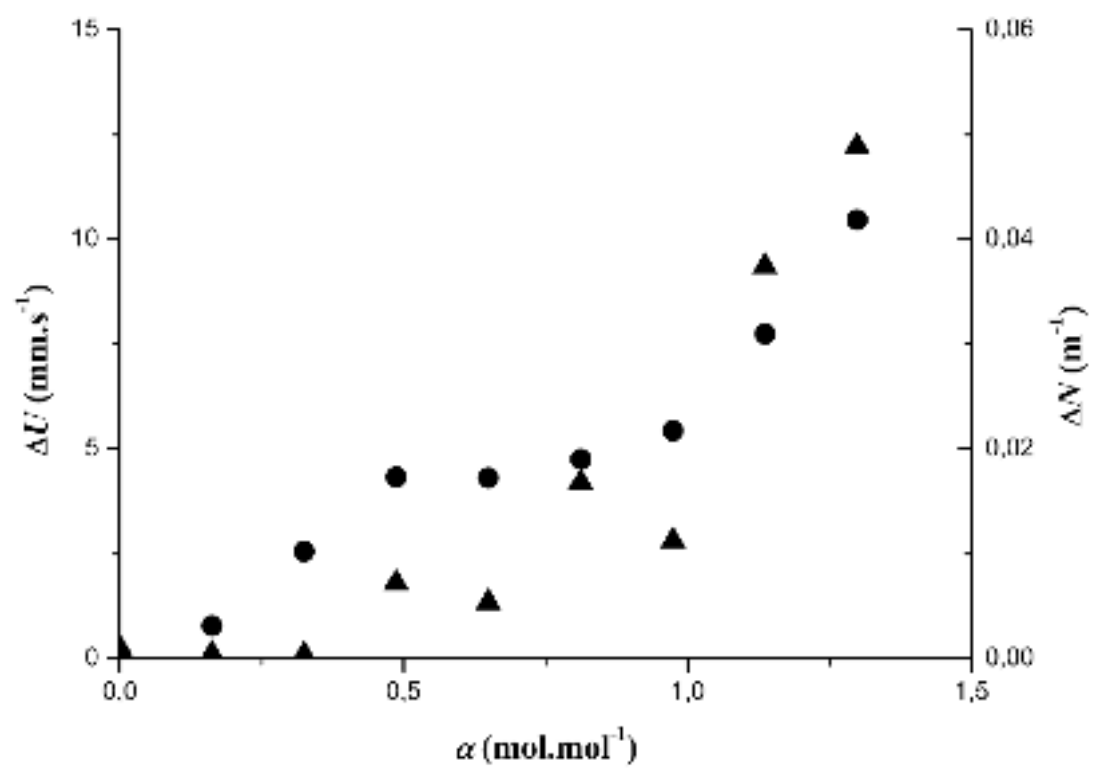

Fig. (4). Results of ultrasound titration of citric acid by $\mathrm{CuCl}_{2}(\Delta U$ - circles, $\Delta N$ - triangles). 


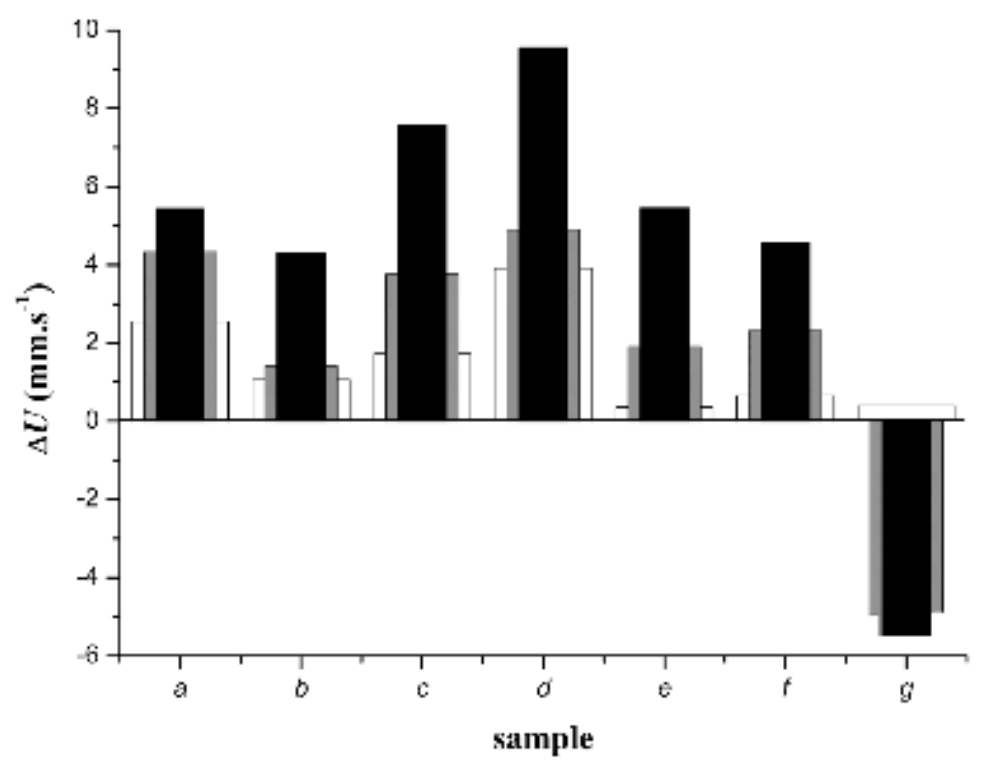

Fig. (5). $\Delta U$ values obtained for three various ratios of added $\mathrm{Cu}^{2+}$ ions and acidic groups in models $(\alpha=0.25-$ white; $\alpha=0.5-$ grey; $\alpha=1.0$ - black): $\mathbf{a}$ - citric acid, $\mathbf{b}$ - phthalic acid, $\mathbf{c}$ - salicylic acid, $\mathbf{d}$ - EDTA, $\mathbf{e}$ - hydroquinone, $\mathbf{f}$ - pyrocatechol, $\mathbf{g}$ - humic acid.

much better complexation agent than others, which is frequently utilized for copper removing from $\mathrm{Cu}$-humic complexes.

Fig. (5) show the measured ultrasound velocities for the used model compounds as well as the humic acid at three various values of $\alpha(0.25,0.5$ and 1$)$. We can see that the highest value of $\Delta U$ was determined for EDTA. This model therefore forms the complexes with very rigid structure corresponding with high stability constant. The difference between EDTA and others models is remarkable mainly at $\alpha$ $=0.25$, where the Cu-EDTA complexes in ratio $1: 1$ are formed. It is interesting that the ultrasound velocities measured for humic acids and hydroquinone are comparable in this stage of titration. While the $\Delta U$-value increases for all used model compounds with further addition of the copper(II) ions, the ultrasound velocity of humic acids decreases. The reason of the $\Delta U$ decrease is described above. Except humic acids the lowest values of $\Delta U$ were determined for models with only one type of functional groups (phthalic acid, hydroquinone, pyrocachol). It is difficult to explain completely the observed differences between the used models and humic acids.

Murray and Linder [8,9] correlated the binding site concentration with the aromaticity of humic acids. hey predicted phthalate (M4, M7) and salicylate (M3, M6) structures as predominant sites for metal ions. As it was mentioned above our sample belongs to highly aromatic humic acids and its degree of aromaticity is $44 \%$ [31]. For this kind of humics the main binding sites corresponding with models M1, M2, M3 and M4 are favourable. With respect to low content of the hydroxylic groups in comparison with the carboxylic ones in lignitic humic acids $[16,17,22]$, mainly structures M1 and M4 are probably formed in the complexation.

Bryan et al., [10] write that the metal binding sites in humic acids are not "pre-formed" entities, but rather, the humic coordination sphere forms as the metal binds, almost certainly involving molecular conformational rearrangement. Similar re-arrangement was observed also in [32] and [33]. The EXAFS study [34] showed that the $\mathrm{Cu}$ distances in the studied humic complexes were shorter that the distances in aqua or salicylate complexes. Results of this work indicate that axial oxygens are more tightly bound with the central cation $\left(\mathrm{Cu}^{2+}\right)$ than those in either aqua or salicylic complexes. This strong binding of copper by humic acids and their ability of conformational changes could be the main reasons of the observed differencies between humic acids and the used models.

\section{CONCLUSIONS}

It was confirmed that the method of ultrasound spectrometry is very sensitive to studied problems of complexation of humic acids and their ,models“ mainly due high resolution of the used device. The measured ultrasound velocity reflects local elasticity and density of material, which are strongly influenced by molecular arrangements, conformation and solvation shell. The concentration dependence of ultrasound velocity enabled to follow the metal interactions with the individual functional groups and to elucidate the effect of structure of complexing molecule on its binding with the metal. The changes in the slope of the concentration dependence were used to find the saturation of binding and to follow the changes of hydration of interacting species. The used model compounds were chosen for their similarity with the active sites in the structure of humic acids. The obtained results confirmed that the interaction of humic compounds with metal ions is the complex problem, which should be studied by various methods to understand better their principles and effects on their function in nature. The method of ultrasound spectrometry is newly utilized in study of the humic complexation. The differences observed for the individual model compounds show that there are active centers not only with various strength and stability of formed complexes but also with their various rigidity and ability of conformational changes. All these properties can 
strongly influence both the immobilization properties (e.g. in soil remediation) and the plant nutrition support of humic acids. The knowledge of structure of binding sites in humic acids could explain their function in nature and help to the utilization of lignite and humic compounds extracted from coals in non-fuel applications.

\section{CONFLICT OF INTEREST}

None declared.

\section{ACKNOWLEDGEMENT}

This work was supported by government funding - Czech Science Foundation, project. Nr. 104/08/0990 and by the project "Centre for Materials Research at FCH BUT" No. CZ.1.05/2.1.00/01.0012 from ERDF.

\section{REFERENCES}

[1] Davies, G.; Ghabbour, E.A.; Cherkasskiy, A.; Fataftah, A. In Humic Substances and Chemical Contaminants; Clapp, C.E.; Hayes, M.H.B.; Senesi, N.; Bloom, P.R.; Jardine, P.M., Eds.; Soil Science Society of America: Anaheim, 2001; pp. 371-395.

[2] Martyniuk, H.; Wieckowska, J. Adsorption of metal ions on humic acids extracted from brown coals. Fuel Process. Technol., 2003, 84, 23-36.

[3] Saar, R.A.; Weber, J.H. Comparison of spectrofluorometry and ionselective electrode potentiometry for determination of complexes between fulvic acid and heavy metal ions. Anal. Chem., 1980, 52, 2095-2100.

[4] Antonelli, M.L.; Calace, N.; Fortini, C.; Petronio, B.M.; Pietroletti, M.; Pusceddu, P. Calorimetric investigation of complex formation of some humic compounds. J. Therm. Anal. Calorim., 2002, 70, 291-297.

[5] Cariati, F.; Erre, L.; Micera, G.; Panzanelli, A.; Piu, P. Interaction of metal-ions with humic-like models. 2. Thermal properties of hexaaquametal (II) bis(2,6-dihydroxybenzoate) dihydrate complexes. Thermochim. Acta, 1983, 66, 1-10.

[6] Micera, G.; Piu, P.; Erre, L.; Cariati, F.; Pusino, A. Interaction of metal ions with humic-like models. 3. Thermal behavior of aquobridged polymeric complexes of 2,6-dimethoxybenzoic acid with divalent metal ions. Thermochim. Acta, 1984, 77, 67-75.

[7] Borges, F.; Guimaraes, C.; Lima, J.L.; Pinto, I.; Reis, S. Potentiometric studies on the complexation of copper(II) by phenolic acids as discrete ligand models of humic substances. Talanta, 2005, 66, 670-673.

[8] Murray, K.; Linder, P.W. Fulvic acids - Structure and metal binding.1. A random molecular model. J. Soil Sci., 1983, 34, 511-523.

[9] Murray, K.; Linder, P.W. Fulvic acids - Structure and metal binding. 2. Predominant metal binding sites. J. Soil Sci., 1984, 35, 217-222.

[10] Bryan, N.D.; Robinson, V.J.; Livens, F.R.; Hesketh, N.; Jones, M.N.; Lead, J.R. Metal-humic interactions: A random structural modelling approach. Geochim. Cosmochim. Acta, 1997, 61, 805-820.

[11] Gregor, J.E.; Powell, H.K.J.; Town, R.M. Evidence for aliphatic mixed mode coordination in copper(II)-fulvic acid complexes. J. Soil Sci., 1989, 40, 661-673.

[12] Baker, H.; Khalili, F. Comparative study of binding strengths and thermodynamic aspects of $\mathrm{Cu}(\mathrm{II})$ and $\mathrm{Ni}(\mathrm{II})$ with humic acid by Schubert's ion-exchange method. Anal. Chim. Acta, 2003, 497, 235-248.

[13] Pandey, A.K.; Pandey, S.D.; Misra, V.; Viswanathan, P.N. Formation of soluble complexes of metals with humic acid and its environmental significance. Chem. Ecol., 1999, 16, 269-282.
[14] Klucakova, M.; Pekar, M.; Valkova, D. In Humic Substances Linking Streucture to Functions, Proceedings of the $13^{\text {th }}$ Meeting of the International Humic Substances Society, vol. 45-II, Karlsruhe, Germany, July 30 - August 4, 2006; Frimmel, F.H.; Abbt-Braun, G., Eds.; Schriftenreihe Bereich Wasserchemie Engler-Bunte-Institut der Universität Karlsruhe: Karlsruhe, Germany, 2006; pp. 893-896.

[15] Klucakova, M.; Pelikan, P.; Lapcik, L.; Lapcikova, B.; Kucerik, J.; Kalab, M. Structure and properties of humic and fulvic acids. I. Properties and reactivity of humic acids and fulvic acids. J. Polym. Mater., 2000, 17, 337-356.

[16] Peuravuori, P.; Zbankova, P.; Pihlaja, K. Aspects of structural features in lignite and lignite humic acids. Fuel Process. Technol., 2006, 87, 829-839.

[17] Klucakova, M.; Pekar, M. Behaviour of partially soluble humic acids in aqueous suspension. Colloid Surface A, 2008, 318, 106-110.

[18] Klucakova, M.; Kucerik, J.; Pekar, M.; Valkova, D. In From Molecular Understanding of Innovative Applications of Humic Substances, Proceedings of the $14^{\text {th }}$ Meeting of International Humic Substances Society, Vol. I., Moscow - St. Petersburg, Russia, September 14-20, 2008; Perminova, I.V.; Kulikova, N.A., Eds.; Humus Sapiens: Moscow, Russia, 2008, pp. 99-102.

[19] Kankia, B.I.; Funck, T.; Uedaira, H.; Buckin, V.A. Volume and compressibility effects in the formation of metal-EDTA complexes. J. Solution Chem., 1997, 26, 877-888.

[20] Kharakoz, D.P.; Sarvazyan, A.P. Hydrational and intrinsic compressibilities of globular proteins. Biopolymers, 1993, 33, 11-26.

[21] Kudryashov, E.; Smyth, C.; O'Driscoll, B.; Buckin, V. Highresolution ultrasonic spectroscopy for analysis of chemical reactions in real time. Spectroscopy, 2003, 18, 26-32.

[22] Klucakova, M.; Pekar, M. In Humic substances: Molecular details and applications in land and water conservation; Ghabbour, E.A.; Davies, G., Eds.; Taylor \& Francis: New York, 2005; pp. 167-188.

[23] Maketon, W.; Ogden, K.L. Synergistic effects of citric acid and polyethyleneimine to remove copper from aqueous solutions. Chemosphere, 2009, 75, 206-211.

[24] Field, T.B.; McCourt, J.L.; McBryde, W.A.E. Composition and stability of iron and copper citrate complexes in aqueous solution. Can. J. Chem., 1974, 52, 3119-3124.

[25] Dunhill, R.H.; Pilbrow, J.R.; Smith, T.D. Electron spin resonance of copper(II) citrate chelates. J. Chem. Phys., 1966, 45, 1474-1481.

[26] Ivanov, S.; Tsakova, V. Influence of copper anion complexes on the incorporation of metal particles in polyaniline - Part I: Copper citrate complex. J. Appl. Electrochem., 2002, 32, 701-707.

[27] Natter, H.; Hempelmann, R. Nanocrystalline copper by pulsed electrodeposition: The effects of organic additives, bath temperature, and pH. J. Phys. Chem., 1996, 100, 19525-19532.

[28] Zaitoun, M.A.; Lin, C.T. Chelating behavior between metal ions and EDTA in sol-gel matrix. J. Phys. Chem. B, 1997, 101, 1857-1860.

[29] Gyliene, O.; Aikaite, J. Formation of binuclear EDTA and $\mathrm{Cu}(\mathrm{II})$ complexes in aqueous solutions. Pol. J. Chem., 2003, 77, 99-104.

[30] Jordan, J.; Alleman, T.G. Thermochemical titrations - enthalpy titrations. Anal. Chem., 1957, 29, 9-13.

[31] Barancikova, G.; Klucakova, M.; Madaras, M.; Makovnikova, J.; Pekar, M. Chemical structure of humic acids isolated from various soil types and lignite. Humic Subst. Environ., 2003, 3, 3-8.

[32] Wershaw, R.L. Application of a membrane model to the sorptive interactions of humic substances. Environ. Health Perspect., 1989, 83, 191-203.

[33] Klucakova, M.; Pekar, M. Study of structure and properties of humic and fulvic acids. III. Study of complexation of $\mathrm{Cu}^{2+}$ ions with humic acids in sols. J. Polym. Mater., 2003, 20, 145-153.

[34] Korshi, G.V.; Frenkel, A.I.; Stern, E.A. EXAFS study of the inner shell structure in copper(II) complexes with humic substances. Environ. Sci. Technol., 1998, 32, 2699-2705. 